\title{
EVALUATION OF OPERATIONAL PROCEDURES FOR USING A TIME-BASED AIRBORNE INTER- ARRIVAL SPACING TOOL
}

\author{
Rosa M. Oseguera-Lohr* \\ Gary W. Lohr \\ Terence S. Abbott* \\ NASA Langley Research Center, Hampton VA \\ Todd M. Eischeid \\ Booz-Allen and Hamilton
}

\begin{abstract}
$\underline{\text { Abstract }}$
An airborne tool has been developed based on the concept of an aircraft maintaining a time-based spacing interval from the preceding aircraft. The Advanced Terminal Area Approach Spacing (ATAAS) tool uses Automatic Dependent Surveillance-Broadcast (ADS-B) aircraft state data to compute a speed command for the ATAAS-equipped aircraft to obtain a required time interval behind another aircraft. The tool and candidate operational procedures were tested in a high-fidelity, full mission simulator with active airline subject pilots flying an arrival scenario using three different modes for speed control. The objectives of this study were to validate the results of a prior Monte Carlo analysis of the ATAAS algorithm and to evaluate the concept from the standpoint of pilot acceptability and workload. Results showed that the aircraft was able to consistently achieve the target spacing interval within one second (the equivalent of approximately $220 \mathrm{ft}$ at a final approach speed of $130 \mathrm{kt}$ ) when the ATAAS speed guidance was autothrottle-coupled, and a slightly greater (4-5 seconds), but consistent interval with the pilot-controlled speed modes. The subject pilots generally rated the workload level with the ATAAS procedure as similar to that with standard procedures, and also rated most aspects of the procedure high in terms of acceptability. Although pilots indicated that the head-down time was higher with ATAAS, the acceptability of head-down time was rated high. Oculometer data indicated slight changes in instrument scan patterns, but no significant change in the amount of time spent looking out the window between the ATAAS procedure versus standard procedures.

\section{Introduction and Background}

In recent years, air travel has increased at unprecedented rates, leading to traffic congestion at many of the nation's busiest terminal areas. With this trend expected to continue into the foreseeable future,

\footnotetext{
* AIAA Member
}

many government and industry efforts have been focused on research programs aimed at alleviating this problem through development of procedures for airborne and ground-based use with supporting new technologies. To address this problem, the National Aeronautics and Space Administration (NASA) Advanced Air Transportation Technologies (AATT) Project has developed the concept of Distributed Air/Ground Air Traffic Management (DAG-TM). The DAG-TM concept involves various levels of collaboration between airborne and ground-based resources to enable less-restricted and more efficient aircraft trajectories throughout all phases of flight, leading to increased airport capacity ${ }^{1}$.

One element of the DAG-TM concept focuses on terminal area operations and requires development of procedures and technologies that allow aircraft to have more flexibility in choosing an efficient route through the terminal area while arriving at the runway threshold properly spaced from the preceding aircraft ${ }^{2}$. One of the goals for capacity enhancements in the terminal area is to safely reduce the excess spacing buffers currently used by controllers when separating aircraft, through reduction in the uncertainty in relative airplane positions. This requires the use of more accurate means of controlling the spacing intervals between arriving aircraft.

Previous research investigated the feasibility of using traffic information displayed on the flight deck to enable airborne-managed spacing ${ }^{3-6}$. Simulator experiments conducted at NASA Langley Research Center involving the use of Cockpit Display of Traffic Information (CDTI), including a display of the lead traffic's location and other predictors on the subject aircraft's Navigation Display found that time-based spacing was the most useful technique. A "time box" was used to represent the position where the subject aircraft ("ownship") should be, and provided a positional target for the ownship to achieve in order to be at the right spacing interval behind the aircraft it was following. The spacing interval was assigned by Air 
Traffic Control (ATC). The studies concluded that this concept was feasible from a crew workload and acceptability standpoint. Accurate knowledge of the positions and speeds of the aircraft with fast update rates are necessary for concept feasibility. Recent improvements in display and computing capabilities and broadcast of traffic state data make the concept more realizable.

Recently, an airborne tool has been developed at NASA Langley that is based on this earlier work, but has been refined to better meet the objectives of the approach spacing concept. This tool, which is called the Advanced Terminal Area Approach Spacing (ATAAS) tool, is based on the concept of an aircraft maintaining a time-based, rather than distance-based, spacing interval from the preceding aircraft ${ }^{7}$. The ATAAS tool uses Automatic Dependent Surveillance-Broadcast (ADS-B) aircraft state data plus final approach speeds and wind data to compute a speed command for the ATAASequipped aircraft to follow in order to maintain the required time interval behind the other aircraft, and has undergone extensive Monte Carlo analysis to characterize and refine its performance. Although the tool has many potential applications in different types of operational scenarios, including en-route and oceanic operations, the concept of in-trail spacing in the terminal area (i.e., aircraft are spacing longitudinally while following directly behind each other) was the logical first step in the evolution of the end-state goal of more efficient and flexible maneuvering through the terminal area.

To develop the concept of in-trail, airborne-managed spacing in the terminal area, a nominal scenario was defined, to include system and operational (crew and controller) procedures, with candidate phraseologies and required crew interface with the ATAAS tool. The concept includes the use of a charted Standard Terminal Arrival Route (STAR), similar to those currently in use today. The arrival route is extended to include a complete lateral path to the runway, plus a vertical profile (speed and altitude) all of which become part of the nominal arrival clearance. The nominal speed profile associated with this charted procedure provides a basis around which the ATAAS algorithm will build the speed commands to be used by the flight crew. The basic system procedure is the issuance of an additional clearance from the controller to the ATAAS-equipped aircraft flight crew, which identifies the traffic to follow and the assigned spacing interval. Theoretically, this clearance could be issued at any time during the arrival. Once the flight crew accepts the spacing clearance and begins following the ATAAS-commanded speeds, no further speed clearances are needed from the Air Traffic Service Provider (ATSP), but other normal communications (frequency changes, approach and landing clearances) take place as expected.

Part of the concept vision is the ability for un-equipped aircraft (i.e., those without an ATAAS implementation) to also participate in this operation, by means of the charted arrival. Including the nominal routing and speed profile as part of the charted arrival allows an aircraft that can maintain the charted profile to be cleared for and fly this arrival. By broadcasting its position and the appropriate data, it can also serve as a lead aircraft for the ATAAS-equipped aircraft sequenced behind it. This concept can also be extended to lower-density facilities as their traffic levels increase. This procedure allows aircraft to perform approach spacing operations into these facilities, enabling more consistent and reliable spacing of arrivals with minimal changes to infrastructure.

The ultimate goal behind the in-trail concept is not to accurately and precisely space individual pairs of aircraft, but rather to achieve a system-wide improvement in performance. This improvement will be realized by obtaining better consistency in spacing from a system-wide standpoint, sometimes at the expense of having excessive spacing between individual aircraft pairs. As such, no single aircraft will be given guidance to aggressively achieve a spacing interval beyond what would normally be expected in current-day operations. It is readily apparent that increasing the speed of one aircraft excessively in order to "close up the gap" with a preceding aircraft would quickly de-stabilize the system by multiplying the effect on the speed required of every aircraft that is intrail, creating increasingly larger gaps and speeds well beyond acceptable levels by today's standards. In order to enforce this ideal, realistic limits were placed on the speed guidance provided by the ATAAS system. Thus, the commanded speed will not exceed $10 \%$ of the nominal (charted) speed for any given segment on the arrival. In future applications, the reduction in system throughput that could result from this type of limitation could be recovered through other methods, such as adjusting the lateral route in a designated maneuvering area.

A fundamental issue that is unchanged from currentday procedures is the responsibility for maintaining separation between aircraft. Under the new scenario, this responsibility remains with the ATSP. With this in mind, the clearance to conduct the approach-spacing operation is then a clearance to follow the ATAAS speeds, since the aircraft is already in the arrival phase. The clearance phraseology used for this study reflects this. 
Appropriate flight crew procedures were developed to allow interaction with the ATAAS tool, with minimal impact to current workload levels. Supporting display elements were developed to provide information to the crew on the mode of operation and the current state of the ATAAS-equipped aircraft ("ownship") relative to the aircraft it is spacing behind (the "lead" aircraft). A trail of "history dots" behind the lead aircraft show its ground track on the ownship's Navigation Display (ND), and can be used instead of an "area navigation" (RNAV) route for lateral navigation. A simple pilot interface with the ATAAS tool is provided to allow the crew to select the lead aircraft and enter other appropriate data.

In order to evaluate workload and pilot acceptability issues, and to explore the feasibility of the operational concept (i.e., can the assigned spacing interval be consistently achieved with the algorithm implemented on real-world equipment), the nominal in-trail arrival scenario was tested in a high-fidelity, full mission simulator with airline subject pilots flying the scenario using various modes for speed control. Aircraft and ATAAS state and mode data were collected, and pilots provided subjective ratings of perceived workload levels and various other aspects of the concept through questionnaires.

\section{$\underline{\text { Scenario }}$}

\section{Method of Test}

The simulated environment for this study was the Memphis International Airport (MEM) and surrounding terminal area. The routing flown for the scenario was the WILDR4 STAR, which was extended to include a downwind and base leg for a transition to the ILS (Instrument Landing System) runway 36 Right final approach course. The same scenario was used for all runs, and began with the aircraft level at $8000 \mathrm{ft}, 250 \mathrm{kt}$ indicated airspeed (IAS), approximately $10 \mathrm{nmi}$ prior to the downwind turn. No wind conditions were simulated. A single subject pilot was used for data collection, with a confederate pilot flying in the right seat. The confederate pilot was a retired airline pilot from a major air carrier, with knowledge of research methods. Since crew interactions were not a focus of this study, this arrangement provided the opportunity to obtain data on acceptability and workload from the subject pilot while still maintaining the realism of operating in a two-person crew, full-mission environment.

\section{$\underline{\text { Simulation environment }}$}

The simulation environment included communications with a simulated ATC facility. Normal radio communications were simulated. Other traffic in the pattern was simulated using pre-recorded tracks of arriving aircraft that were played back during the scenario. The traffic level corresponded to what might be expected at a busy terminal area. The traffic appeared on the ND as TCAS (Traffic Alert / Collision Avoidance System) targets, using symbology very similar to what pilots use in current operations.

A single stream of arriving traffic was simulated and used for all runs (i.e., call signs of the aircraft in the arrival stream were the same for all runs). The subject aircraft (call sign "NASA 557") was number 8 in trail to the runway at the start of the scenario. The traffic aircraft (call sign UAL903) immediately ahead of NASA557, and all other aircraft in the scenario followed the nominal charted speeds in an orderly manner, with no unusual or rapid changes in speed.

\section{Crew Procedures}

The subject pilot was briefed on the crew procedures upon arrival, to supplement a copy of the flight manual bulletin and charts, which were developed for the study and previously mailed to the subject pilot. Included in the bulletin was background information on the operation and the charted procedure, a summary of the procedures for interacting with the custom ATAAS Flight Management Computer (FMC) Control-Display Unit (CDU) pages, and a checklist indicating the crewmember responsibilities for each step in the operation. To summarize, the Pilot Not Flying (PNF) was responsible for selecting the assigned traffic to follow using the ATAAS CDU pages, entering the assigned spacing interval and any other necessary data (such as final approach speeds), and acknowledging the clearance with ATC. The Pilot Flying (PF) was responsible for activating the ATAAS system and following the speed guidance. Both pilots were responsible for monitoring of speed and other cues to ensure compliance with the speed guidance. These tasks were to be integrated with other normal duties.

The approach spacing clearance was issued after the aircraft had turned onto the downwind leg and switched frequencies. The approach clearance was a separate clearance, issued when the aircraft was on base leg. The assigned lead aircraft (traffic to follow) and spacing interval in the clearance were the same for all runs.

\section{$\underline{\text { ATAAS Interface }}$ EADI Display}

Output from the ATAAS system was shown in various locations and forms on the pilots' displays. Pilots obtained ATAAS guidance from these displays, and 
additional data on status from the FMC CDU pages (also described below). The ATAAS symbology on both Electronic Attitude Director/Indicator (EADI) and ND appeared only after a lead aircraft and spacing interval were selected from the CDU page.

The EADI used for this experiment was the standard B757 EADI, very similar to those currently in use in most aircraft of this type (see Figure 1). It includes a Fast/Slow (F/S) Indicator on the left side of the display, which normally is tied to the speed mode being used. For example, when the crew is flying the aircraft in "Speed" mode (meaning speed is controlled by dialing the target speed into the Mode Control Panel (MCP) Speed window), the red "speed bug" on the airspeed indicator moves to the target speed displayed in the window, and the F/S indicator reflects the relationship of the current aircraft speed with the target speed. If the current speed is faster than the target speed in the MCP window, the pointer on the $\mathrm{F} / \mathrm{S}$ indicator moves towards the "F"; if the current speed is slower than the MCP window speed, the pointer moves towards the "S".

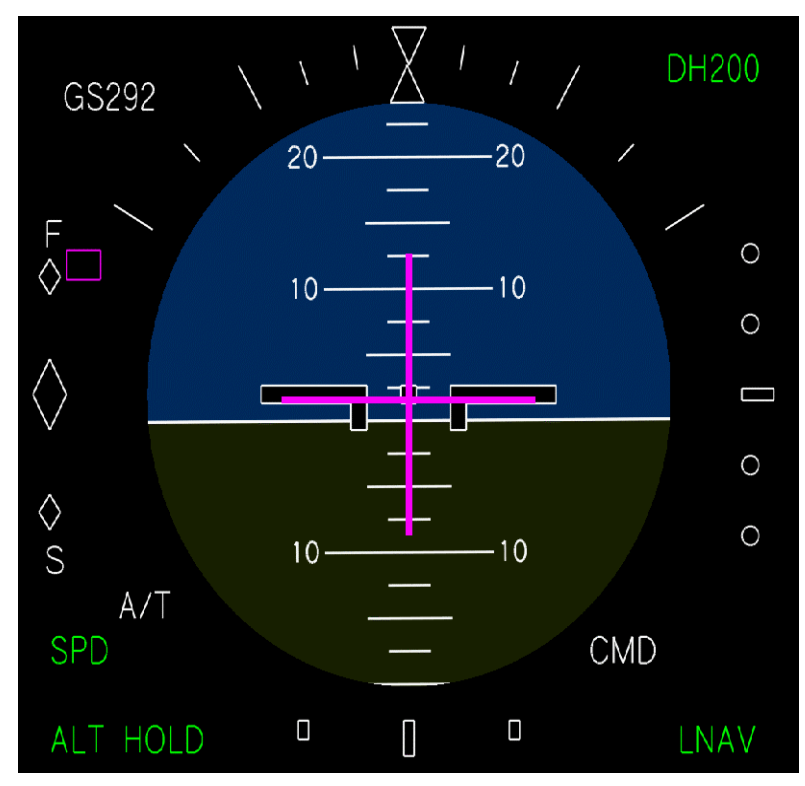

Figure 1. EADI with normal symbology.

The ATAAS implementation on the EADI (see Figure 2) made use of the F/S indicator to reflect the relationship between the current aircraft speed and the ATAAS speed (the speed guidance output from the ATAAS algorithm). The airspeed "bug" on the electromechanical airspeed indicator also tracked the ATAAS speed guidance, giving the pilots another reference. In addition, the commanded speed appeared in digital form above the F/S Indicator, in green font. This display differed slightly from what was shown by the F/S Indicator in that it represented the end-point of the speed change, rather than the instantaneous ATAAS speed. For example, when the ATAAS guidance commanded a speed reduction from 210 knots to 170 knots, the speed annunciated above the F/S Indicator changed from " 210 " to " 170 " to inform the pilot of the endpoint of the speed reduction, but the pointer on the F/S Indicator followed a scheduled speed reduction, as did the bug on the airspeed indicator.

A 5-kt threshold for annunciating a new commanded speed (above the F/S Indicator) was used. When a speed change occurred, the new commanded speed appeared with a box around it that flashed for several seconds to get the pilot's attention.

A feature of the ATAAS algorithm is its ability to provide a smooth transition from the commanded speed required for achieving the spacing interval, to the final approach speed entered on the ATAAS Approach Data CDU page. The algorithm is automatically switched to this approach mode near the final approach fix to allow enough time to achieve a stabilized final approach. When the algorithm transitions to this mode, it is no longer actively "spacing" on the lead aircraft, and the commanded speed annunciation changes from the numeric value to "APPR" to inform the pilot of the change.

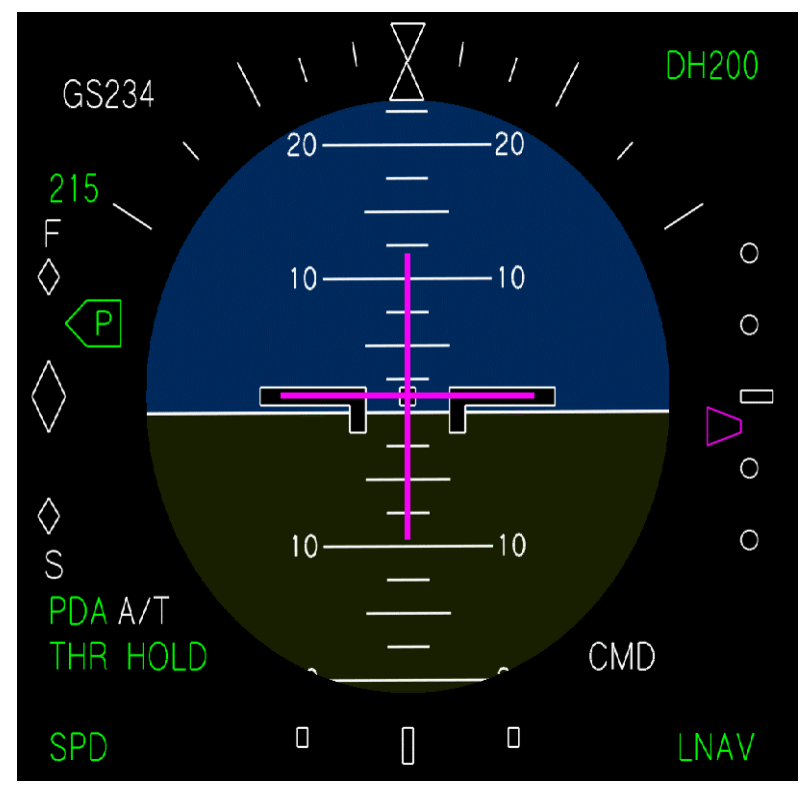

Figure 2. EADI with ATAAS symbology

\section{Navigation Display}

Symbology added to the ND provided additional information on the ATAAS guidance and aircraft spacing status (see Figure 3). There were three main 
pieces of information that were provided: 1) a data block that included currently entered ATAAS data and lead aircraft range; 2) spacing position indicator; and 3) lead aircraft history dots.

The data block showed the desired spacing interval in seconds (the interval entered in the CDU approach spacing page), the call sign of lead aircraft (the aircraft selected on the CDU page), and the current distance in nmi to the lead aircraft. The data block updated as the distance between the aircraft changed, and to reflect any other changes (such as if the pilots selected a new lead aircraft or spacing interval). In addition to these pieces of information, the commanded speed also appeared as the top line on the data block. This commanded speed was identical to the one that appeared above the F/S Indicator on the EADI.

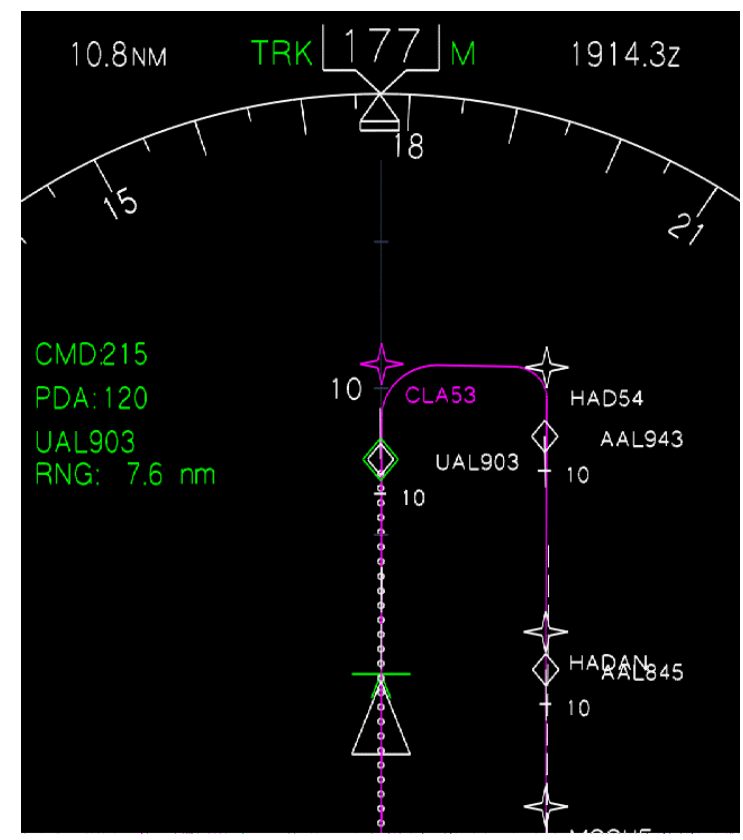

Figure 3. ND with ATAAS symbology

A spacing position indicator was provided to show the position where the ownship needed to be in order to achieve the proper spacing interval (based on currently entered lead aircraft and desired spacing interval). This indicator consisted of a short green line perpendicular to the ownship's ground track, with an inverted " $\mathrm{V}$ " attached to the midpoint of the line. When the ownship was properly spaced, the spacing position indicator fit exactly over the apex of the white triangular ownship symbol. If the spacing position indicator was behind the apex of the ownship symbol, it meant that the ownship was ahead of where it should be (actual spacing interval was less than the targeted interval). Conversely, if the spacing position indicator was ahead of the ownship symbol, it meant that the ownship was behind where it should be (actual spacing interval was greater than the targeted interval). This indicator provided a visual reference of the ownship's position relative to the desired spacing interval.

The lead aircraft history dots showed the ground track of the currently selected lead aircraft. When an aircraft was initially selected as the lead, its history dots appeared and instantly extended backwards past the ownship's current position, depicting its ground track. This feature allows an ATAAS-equipped aircraft to maintain spacing behind an aircraft that is not on an RNAV route, such as one that is on a visual approach or being radar-vectored, by following its history dots.

\section{FMC-CDU pages}

Interface with the ATAAS system was accomplished through customized FMC-CDU pages, accessed through a function key on the CDU, which was labeled "ATC". When this key was pushed, the Approach Spacing page appeared, with a list of the other aircraft in the terminal area on the right side of the page, in alphabetical order by call sign (see Figure 4). When the pilot line-selected the call sign of the aircraft in front of him, a block of data appeared on the left side of the page, showing the current actual spacing interval, the current actual distance between the lead and ownship, and the lead aircraft's groundspeed. These data provided the pilot a method to check the actual spacing against what was assigned, ensuring upon initialization of the system that there was agreement within some reasonable level (plus or minus 20 seconds). A prompt for entering the desired spacing interval appeared on Line Select Key 2 Right (LSK2R) on this page. Figure 5 shows what this page looked like after selecting the aircraft and entering the spacing interval.

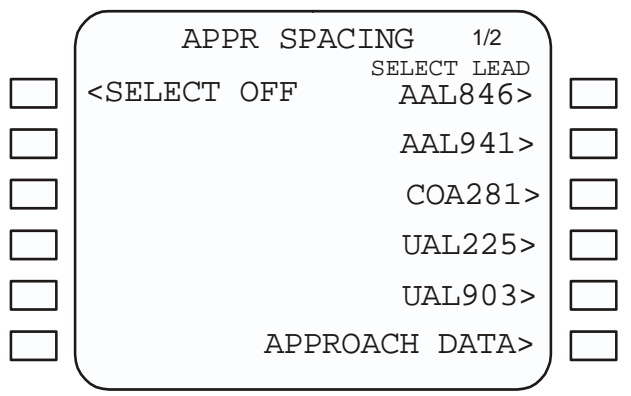

Figure 4. CDU Approach Spacing Page before lead aircraft selection

At the bottom of the CDU screen on this page was a prompt on LSK6R that was labeled Approach Data. Line-selecting this key took the pilot to the Approach Data page, which contained prompts for entering other 
relevant parameters, such as final approach speed (FAS) for the ownship and the selected lead aircraft, minimum distance (if something other than wakevortex separation is desired), and airport surface winds. For this experiment, data entry was only required for the final approach speeds, since the simulated environment was a calm day, and the standard separation was used.

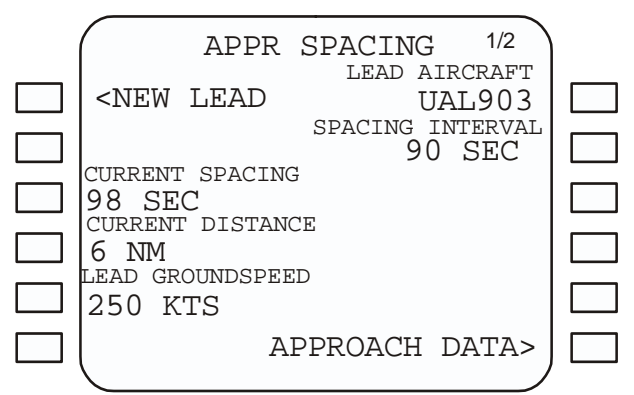

Figure 5. CDU Approach Spacing Page after lead aircraft selected and time interval entered.

Facilities

$$
\text { Flight simulator }
$$

The facility used for this experiment was the Integration Flight Deck (IFD) simulator, a full-workload Boeing 757 research flight simulator, operating in fixed-base mode. This simulator emulates the flight deck of the NASA Langley ARIES (Airborne Research Integrated Experiments System), which is a modified B757-200 aircraft used for research flights. Because it is used for research, there are some non-standard components that are used to support the experimental systems. The main difference, for the subject pilots, involved the location of the control panel for the ND, which is located further back on the aisle stand than in the standard configuration. This panel also contained the pushswitch that was used to activate the ATAAS system after the lead aircraft and spacing interval were entered.

\section{$\underline{\text { ATC station }}$}

Because the focus of this study was on the pilot acceptability and workload issues, minimal effort was spent on developing a ground-side capability to support the ATC environment. A station located remotely from the simulator cab provided the controller with a display of the air traffic and various other pieces of information. A single Air Traffic Controller operated the station and provided the real-time communications with other simulated traffic and the IFD cab. Communications were provided through the pilots' headsets in the simulator cab to simulate radio communications.
A realistic communications environment was created through the activation of pre-recorded sound files, which simulated transmissions from the other aircraft in the terminal area. The controller could activate the appropriate sound file after issuing a clearance to an aircraft to simulate a response from that aircraft. Communications with the subject aircraft (simulator) were scripted, with a pre-determined spacing interval used for all the runs.

\section{$\underline{\text { Subject pilots }}$}

Eight subject pilots were used for data collection. The pilots were required to be type-rated and current in the B757 aircraft. Total flight hours for each pilot ranged between 4000 and 17000. Two pilots had between 3001000 hours in type, and the remainder had over 1000 hours in type. There were five first officers and three captains, from a total of four different airlines.

\section{Data collection}

Aircraft state data were obtained from the simulator, and included position, altitude time-histories, and many other parameters relating to the mode of operation of the autoflight system. Data from the ATAAS system included the state in which the system was operating, in addition to the commanded speed and the time interval and distance between the ownship and lead aircraft.

Subjective data were obtained by giving the pilots questionnaires on which their opinions were solicited in several ways. Two structured questionnaires were administered, one following completion of each ATAAS run (the Post-Run Questionnaire), and one at the end of the day, after completing all the runs (the Post-Test Questionnaire). In addition, the pilots completed a self-assessment of workload following each run, using the NASA TLX Task-Load Index method $^{8}$.

\section{Test schedule}

Each pilot was scheduled for one complete day of test. The morning was spent in training, with a verbal briefing followed by simulator training. The data runs were completed in the afternoon.

For simulator training, each pilot was given a short verbal briefing by the confederate pilot upon entering the simulator cab. This briefing covered various details such as differences between the simulator cab and a typical B757 aircraft. The pilots were then required to fly three complete runs for training, one with each of 
the different modes of speed control used for following the ATAAS guidance.

The eight data runs (see Table 1) were then completed, with each data run lasting approximately 20 minutes. Calibration of the eye-tracker and completion of NASA TLX and Post-Run questionnaires following each run took about another 10 minutes. Following completion of the last run, the subject pilot was taken back to the briefing room to complete the Post-Test questionnaire.

Table 1. Test Matrix

\begin{tabular}{|l|c|c|}
\hline Subject Role> & $\begin{array}{l}\text { Pilot } \\
\text { Flying }\end{array}$ & $\begin{array}{l}\text { Pilot Not } \\
\text { Flying }\end{array}$ \\
\hline Baseline & 1 & 2 \\
\hline MCP speed select & 3 & 4 \\
\hline Manual Throttle & 5 & 6 \\
\hline Autothrottle coupled & 7 & 8 \\
\hline
\end{tabular}

$\underline{\text { Results }}$

One of the goals of this study was to validate the results from the previous Monte Carlo analyses in terms of the algorithm's ability to provide speed guidance to deliver the aircraft at the assigned spacing interval. Obviously this ability is also dependent on the manner in which the guidance is followed, and it was recognized that this could have an effect on the spacing interval ultimately achieved. The speed guidance provided by the ATAAS system did not differ in any way for any of the ATAAS scenarios, and was not dependent on the mode of speed control used. The difference between the ATAAS scenarios was only in the way the pilots controlled the speed.

In the baseline scenario, the goal was not to try to achieve the same or similar results for inter-arrival spacing, since the controller's ability to space aircraft was not relevant. The baseline scenario was flown to provide the pilots a comparison for workload and acceptability ratings.

\section{$\underline{\text { Arrival Time Interval }}$}

In general terms, the ATAAS guidance provided a means for achieving a target threshold arrival interval within +/- 5 seconds (this equates to approximately $1100 \mathrm{ft}$ at the approach speed of $130 \mathrm{kt}$ ) across all conditions. Whether or not this performance level is adequate is the subject of another study, and most likely depends on the specific traffic conditions and what level of performance a facility is trying to achieve. More specifically, for this study it was found that the best results (+/- $1 \mathrm{sec}$, equivalent to $220 \mathrm{ft}$ ) were achieved with close speed control to follow the ATAAS guidance. For comparison purposes, a simulator study conducted at Langley in 1990 using conventional air traffic control methods and ground-based automation resulted in a delivery precision on the order of approximately 12 seconds 9 .

Figure 6 shows the resulting mean intervals at the runway threshold for all the ATAAS runs. They are divided by mode of speed control: Manual (pilot controlled speed by manually moving throttle levers, autothrottles off), MCP (pilot controlled speed from MCP speed window), and autothrottle-coupled (speed controlled by autothrottles coupled with ATAAS guidance). The desired interval was the same for all runs (90 seconds). As can be seen in this figure, the autothrottle-coupled condition resulted in the most accurate spacing, with mean intervals within one second of the target. This was true for both the Pilot Flying (PF) and Pilot Not Flying (PNF) positions. As expected, whether the subject or confederate pilot was flying, the results were approximately equivalent.

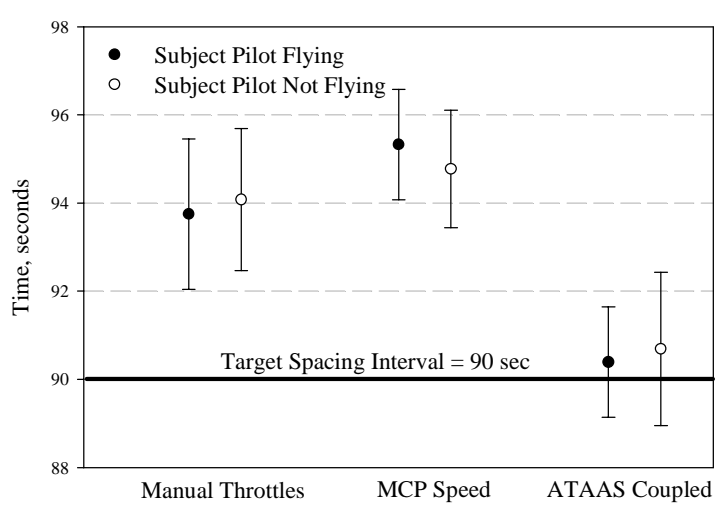

Figure 6. Spacing Interval at Runway Threshold

The manual throttle condition resulted in a mean error of approximately four seconds late, and the MCP condition in a mean error of approximately five seconds late. Standard deviations for these conditions were comparable with the autothrottle-coupled condition, both for PF and PNF positions.

These results are consistent with expectations. In the autothrottle-coupled condition, the aircraft is being controlled closely to the ATAAS speed guidance output, so it is expected that it would result in an interval close to the target. In the other two cases, the speed is being controlled by the pilot following the ATAAS guidance, and is subject to how closely the speed profile is followed. This can be especially crucial in the final approach segment, where differences in the final approach speed decelerations can affect the arrival time at the threshold and cannot be corrected because 
the trailing aircraft is no longer actively spacing on the lead aircraft. In other words, if the pilot slows the airplane faster than the ATAAS speed profile prediction, the aircraft will take longer to cover the same distance because it is at a slightly slower speed for that segment of the approach. The effect is on the order of a few seconds, but if the user is trying to achieve an arrival time within $+/-5$ seconds (as an example), then this small amount of speed difference can affect the result. This is most likely a display or training issue, which results in the pilots not closely flying the ATAAS speed schedule.

\section{$\underline{\text { Pilot interaction with guidance }}$}

A parameter of interest in evaluating the system's performance from a pilot acceptability standpoint was the number of times during each run that the commanded speed changed. This could give an indication of the additional workload required when working with the ATAAS system, since the pilots were required to react to any changes in commanded speed in a similar manner to a new speed clearance from ATC. For the segment in question (starting from being established on the downwind leg through final approach), a pilot would nominally be issued two or more speed changes by ATC, in addition to the pilotinitiated deceleration to the final approach speed at the final approach fix. For comparison, the data summarized in Table 2 reflect the number of times that the annunciated ATAAS speed command changed through the duration of each run. These data were obtained from videotape recordings of the primary instruments (EADI and ND) during the data runs. An annunciated speed command was not counted until it had remained steady for a period of 15 seconds or more when the system was initially activated, and subsequent speed changes were not counted if they happened less than 5 seconds apart. This latter situation happened only twice, and these changes were counted as one, since the pilot reacted to it as only one speed change.

Table 2. Number of commanded speed changes.

\begin{tabular}{|l|l|l|}
\hline & Mean & Std. Dev. \\
\hline PF Manual & 7.3 & 1.3 \\
\hline PF MCP & 7.5 & 1.5 \\
\hline PF Auto & 5.4 & 0.6 \\
\hline PNF Manual & 6.4 & 1.3 \\
\hline PNF MCP & 6.6 & 1.7 \\
\hline PNF Auto & 5.0 & 0.4 \\
\hline
\end{tabular}

The pilots made more speed changes with ATAAS than in the current environment. However, by making more frequent, but smaller speed changes, the ATAAS algorithm is able to better fine-tune the inter-arrival spacing, while maintaining a very acceptable workload level (as judged by the subject pilots in workload ratings).

Comparing the conditions in Table 2 across PF and PNF roles reveals another interesting pattern. For the MCP and Manual cases, when the subject pilot acted as $\mathrm{PNF}$, there were fewer required changes in commanded speed observed. This could be attributed to the confederate pilot reacting in a manner that resulted in fewer required speed changes, due to his being more familiar with the system's operation and being able to follow the speed guidance more closely. It should be noted, however, that the confederate pilot had a few weeks more experience using the ATAAS system than the subject pilots, but was not trained any differently in its operation. In other words, his performance level might be expected from a pilot after having used the ATAAS system on a more frequent, or daily basis for a period of a few weeks, and having had more of a chance to observe the system's behavior.

\section{$\underline{\text { Workload Results }}$ $\underline{\text { TLX Workload Estimates }}$}

Following each trial, NASA TLX scores were obtained from each pilot. The TLX measure was a composite created by summing the six TLX scale values for each participant. The composite score was used because the individual TLX scales were highly correlated with one another and thus provided redundant information. A 2x4 [(pilot position: flying, not flying) x (mode: baseline, automatic, mode control panel, manual)] within subjects ANOVA (Analysis of Variance) on the TLX workload estimates showed a significant difference in reported workload for pilot position, $F(1$, $7)=7.80, p<.05$. As expected, flying the aircraft $(M=14.68, S D=10.55)$ elicited higher workload estimates than not flying the aircraft $(M=9.60$, $S D=6.04)$. No significant differences were found for mode, $F(3,21)=1.82, p>.05$. Thus, none of the modes used differed significantly from the baseline workload estimates (see Figure 7). This result indicates that for this group of pilots, using the ATAAS system did not add a significant level of workload (over standard procedures). The speed control modes used for following the ATAAS guidance covered three different levels of pilot involvement, from none (autothrottlecoupled) to complete (manual throttle). Even with this additional piloting task, plus the additional CDU interface required for setting up the ATAAS system, the workload level is still well within acceptable levels. 


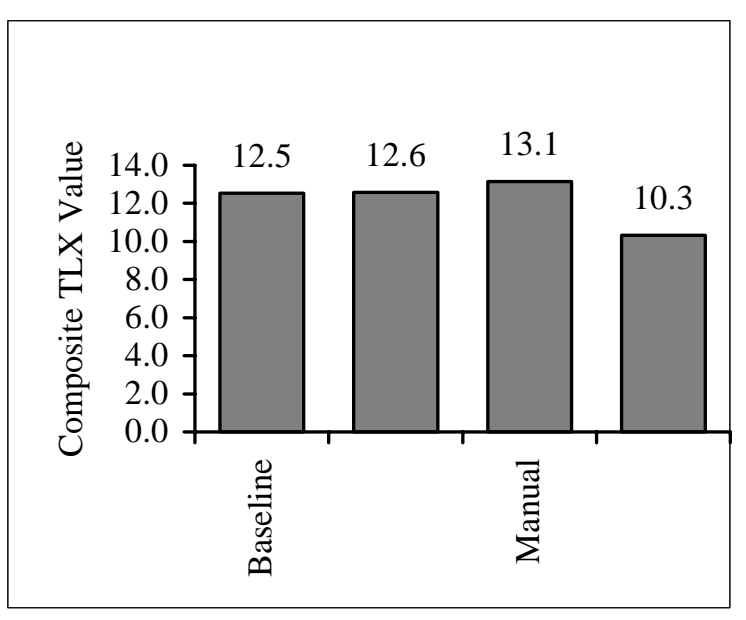

Figure 7. Mean TLX Workload Estimates for Each Mode

Acceptability

\section{Overall Acceptability}

Acceptability ratings for the ATAAS tool were examined separately for downwind, base, and final approach segments using a 7 -point scale (1=not at all acceptable, 4 = borderline, $7=$ very acceptable). Participants responded to the question, "How acceptable was the ATAAS tool during the following phases of the approach procedure?" A separate $2 \times 3$ [(pilot position: flying, not flying) by (mode: automatic, mode control panel, manual] within subjects ANOVA was performed on each approach segment and showed no significant differences for any of the effects. The means and standard deviations for overall ratings of acceptability for the ATAAS tool, across all conditions, were quite high as can be seen in Table 3 . These results indicated an acceptability of the ATAAS tool.

Table 3. Mean overall acceptability of ATAAS tool across all conditions.

\begin{tabular}{|r|r|r|}
\hline Part of Approach & Mean & Std. Dev. \\
\hline Downwind & 6.38 & .96 \\
\hline Base & 6.62 & .53 \\
\hline Final & 6.49 & .66 \\
\hline
\end{tabular}

\section{Acceptability of Head-Down Time}

The acceptability of the amount of head-down time was examined separately for downwind, base, and final segments of approach using a 7-point scale (1=not at all acceptable, 4 = borderline, $7=$ very acceptable).

Participants responded to the question, "How acceptable was the amount of head-down time for the following phases of the approach procedure?" A $2 \times 3$ [(pilot position: flying, not flying) by (mode: automatic, mode control panel, manual] within subjects ANOVA showed no significant differences for any of the effects for any of the phases of approach (downwind, base, final). Incidentally, participants across all conditions rated the amount of head-down time quite acceptable; Table 4 shows the overall means.

Table 4. Mean acceptability of head-down time across all conditions

\begin{tabular}{|r|r|r|}
\hline Part of Approach & Mean & Std. Dev. \\
\hline Downwind & 6.00 & 1.37 \\
\hline Base & 6.23 & 1.07 \\
\hline Final & 6.38 & .80 \\
\hline
\end{tabular}

\section{Confidence with the ATAAS tool}

Confidence ratings with the ATAAS tool's guidance were also examined using a 7 -point scale $(1=$ not at all confident, 4 = borderline, and $7=$ very confident). Participants responded to the question, "How confident were you with the guidance provided by the ATAAS tool during the following phases of the approach procedure?" A 2x3 [(pilot position: flying, not flying) by (mode: automatic, mode control panel, manual] within subjects ANOVA on the confidence ratings was performed on each phase of approach (downwind, base, final). No significant effects were found in any of the three ANOVA's. Participants across all conditions rated their level of confidence quite high for all segments (see Table 5).

Table 5. Mean confidence in guidance provided by ATAAS tool, all conditions

\begin{tabular}{|r|r|r|}
\hline Part of Approach & Mean & Std. Dev. \\
\hline Downwind & 6.35 & 1.06 \\
\hline Base & 6.64 & .57 \\
\hline Final & 6.62 & .58 \\
\hline
\end{tabular}

It should be noted that statistical power in all of the above analyses was too low to detect any true differences among the conditions.

\section{$\underline{\text { Post- Test Questionnaire }}$}

The Post-Test Questionnaire was divided into sections as follows: Acceptability, Procedures, Flight Management Computer-Control/Display Unit, Navigation Display, Electronic Attitude Director Indicator, Training, and Comments. Selected questions from each of the sections are included in the discussion to give a picture of the overall pilot opinions. The questions that required selecting a rating from a scale all used a seven-point scale, where the descriptors for the mid-point (4) indicated neutral conditions (i.e., borderline, unchanged, or moderate). Descriptors for the high end of the scale (7) indicated the more 
desirable condition (i.e., completely effective, completely comfortable, extremely clear, very easy), and for the low end (1) were the more undesirable condition (i.e., completely ineffective, completely uncomfortable, extremely unclear, very difficult). The pilots were also free to insert comments to help clarify their responses. In addition to these questions, a number of open-ended, essay-type questions were asked. The more common or significant pilot responses to these questions are also summarized in the discussion.

\section{Acceptability}

The pilots rated the concept highly in all the questions in the Acceptability section. Generally, they answered that they were comfortable using the ATAAS tool to maintain the assigned spacing in the terminal area, that it was effective in helping maintain the assigned spacing, and that it provided a level of safety better than standard approach procedures. The pilots also responded that the approach spacing procedures and tools could be effectively integrated into the current flight deck operational environment, and rated highly their confidence in the speed guidance provided by the tool.

The subject pilots were also asked if they could think of any real-world situations where the ATAAS procedures and tools might not be effective. The most common responses were adverse weather conditions that required deviations, and gusty wind conditions. Two of the pilots mentioned variations in speed control of the lead aircraft possibly causing a ripple effect on all of the following aircraft, and one pilot mentioned the airspace limitations as a potential problem. One pilot also mentioned as a possible problem a "last minute" insertion of an aircraft into the flow.

\section{$\underline{\text { Procedures }}$}

The pilots rated highly the overall procedure for using the ATAAS guidance system, as well as the clarity of the phraseology used for the approach spacing clearance. All the pilots rated highly how comfortable they were accepting the assigned spacing interval. When asked to compare the amount of time spent looking at displays for the approach spacing procedure with the amount of time spent looking at displays with standard procedures, the responses clearly indicated that it was higher for the approach spacing procedure, although no one made any comment about this being unacceptable.

\section{$\underline{\text { Training }}$}

The subject pilots rated highly the effectiveness of the training received, and indicated that they did not think ATAAS training could be accomplished with only paper or video instructions, or without practice in a high fidelity simulator. When asked specifically what would be the minimum training needed to accomplish the task, most of the pilots indicated video or computer-based (interactive) training at a minimum, more than half also said they would include fixed-base or procedures simulator training.

\section{$\underline{\text { Displays }}$}

Specific questions regarding symbology on the Navigation Display and EADI revealed no major objections or problems; clutter was not considered to be a problem, and although some parts of the symbology were used less than others, no recommendations for major modifications were made.

\section{$\underline{\text { Concluding Remarks }}$}

A concept for providing airborne-managed in-trail spacing in the terminal area was developed, and subsequently evaluated in a full-workload simulator with airline subject pilots. This concept included procedures for flight crew interaction with air traffic controllers as well as with the onboard algorithm that provides speed guidance for achieving the target spacing. Three methods of speed control were evaluated, in addition to a baseline case in which current-day procedures were used.

The main objectives of this study were to validate the results seen in Monte Carlo analyses of the ATAAS algorithm, and to evaluate the concept from the standpoint of pilot acceptability and workload. On the first point, objective data showed the aircraft was able to consistently achieve the target spacing interval within a small standard deviation when the ATAAS speed guidance was autothrottle-coupled. With speed controlled by Mode Control Panel or manual throttle inputs, the mean spacing interval was slightly greater, but the consistency (standard deviation) was on the same order as with autothrottle-coupled. This was most likely a display or training issue, which resulted in the pilots not following the ATAAS speed guidance closely. Although the algorithm is able to compensate for wind effects, no winds were used for this study.

With regard to the second study objective, the subject pilots generally rated the workload level with the ATAAS procedure as similar to that with standard procedures, and also rated most aspects of the 
procedure high in terms of acceptability. Although pilots indicated that the amount of time spent looking at instruments (head-down time) was higher with

ATAAS, the acceptability of head-down time was rated high. Oculometer data obtained from the subject pilots indicated slight changes in instrument scan patterns, but no reduction in the amount of time spent looking out the window (a concern with terminal area operations). In fact, the amount of time spent looking out the window was not significantly changed when pilots used the ATAAS procedure versus the standard procedure.

Although this sample of subject pilots was relatively limited, some important conclusions can be drawn from this study:

- Consistent airborne-managed approach spacing is easily achievable with the ATAAS tool used on real-world equipment.

- Use of simple pilot and controller procedures to accompany the tool can result in a highly acceptable system from the pilot's standpoint.

- Proper training, including fixed-base simulator time is necessary in order to provide pilots with the knowledge and capabilities needed to perform this type of procedure.

- Use of this tool can result in slight changes to the pilots' scan patterns, however a welldesigned interface can minimize the amount of head-down time needed to interact with the tool.

Recommendations for further improvements to the ATAAS tool and procedure:

- Implement with a data link interface for receiving clearance data (lead aircraft ID and final approach speed, spacing interval), to help simplify necessary interactions with the automation.

- Addition of wind data to the ADS-B message to support more accuracy and consistency of the algorithm's performance in the presence of changing winds.

- Addition of a capability to merge aircraft from different arrival streams into a single stream.

\section{$\underline{\text { References }}$}

[1] NASA Advanced Air Transportation Technologies Project: Concept Definition for Distributed Air/Ground Traffic Management (DAG-TM), Version 1.0, September 1999.
[2] Sorensen, John A., "Detailed Description for CE-11, Terminal Arrival: Self Spacing for Merging and In-trail Separation," November, 2000.

[3] Williams, David H., "Time-Based Self-Spacing Techniques Using Cockpit Display of Traffic Information During Approach to Landing in a Terminal Area Vectoring Environment," NASA/TM-84601, April 1983.

[4] Williams, David H. and Douglas C. Wells, "Jet Transport Flight Operations Using Cockpit Display of Traffic Information During Instrument Meteorological Conditions, Simulation Evaluation," NASA/TP-2567, May 1986.

[5] Sorensen, John A., Walter Hollister, Malcolm Burgess, and Dean Davis, "Traffic Alert and Collision Avoidance System (TCAS) - Cockpit Display of Traffic Information (CDTI) Investigation, Phase I Feasibility Study,” DOT/FAA/RD-91/8, April 1991.

[6] Abbott, Terence S., "A Compensatory Algorithm For the Slow-Down Effect on Constant-TimeSeparation Approaches," NASA/TM-4285, September 1991.

[7] Abbott, Terence S., "Speed Control Law For Precision Terminal Area In-Trail Self-Spacing," NASA/TM-2002-211742, July 2002.

[8] Hart, S. G. \& Staveland, L. E. (1988), "Development of a Multi-Dimensional Workload Rating Scale: Results of Empirical and Theoretical Research," P. A. Hancock \& N. Meshkati (Eds.), Human Mental Workload, 139-183. Amsterdam, The Netherlands: Elsevier.

[9] Credeur, Leonard, Jacob A. Houck, William R. Capron, and Gary W. Lohr, "Delivery Performance of Conventional Aircraft by Terminal Area, Time-Based Air Traffic Control, A Real-time Simulation Evaluation,” NASA/TP-2978, 1990. 